\title{
The mysterious origins of coronary vessels
}

\author{
Cell Research (2013) 23:1063-1064. doi:10.1038/cr.2013.90; published online 9 July 2013
}

The origin of the coronary vessels remains a mystery. Here we discuss recent studies that address this puzzle, including new work by Tian et al. recently published in Cell Research.

We face a growing epidemic of coronary vascular disease. Better understanding of the development of this unique vascular system will allow development of new treatment strategies. The origin of the coronary vessels has been a longstanding mystery. Classical anatomists proposed several potential sources for coronary vessels: the proepicardium (PE), the liver, the sinus venosus (SV) and the endocardium (Figure 1). Several recent reports have used sophisticated molecular and cell biological approaches to address this mystery, but have come to apparently contradictory conclusions. Tian et al. [1] use new lineage-tracing approaches to solve this puzzle, leading to new insights and new questions.

Initial studies in avian embryos, based on clonal retroviral labeling, dye labeling and quail-chick interspecies chimeras, indicated that coronary vascular smooth muscle and endothelial cells (vSMCs and ECs) derive from extracardiac sources. Most studies pinpointed the PE, a transient embryonic outgrowth of the septum transversum, as the cell source [2]. PE cells transit to the heart, where they undergo an epithelial to mesenchymal transition (EMT). Based on these data, the predominant view from the early 1990s through the mid-2000s was that coronary vessels formed through a vasculogenic process from PE-derived mesenchymal cells. However, not all studies were in agreement. For example, Poelmann et al. [3] reached a different conclusion and identified the nearby liver primoridium as the cell source. This study concluded that ECs and precursors formed small vessels that initially connected to the SV and then to subepicardial cells overlying the myocardium, which subsequently penetrated the myocardium to form the coronary vessels.

The mainstream view of coronary artery formation from PE-derived ECs has been re-evaluated over the past decade through the use of Cre-LoxP genetic lineage-tracing approaches in mice [4-7]. Several different mouse Cre lines that label populations within the PE were developed. Although these lines generally robustly label coronary vSMCs, they label a low fraction of coronary ECs (generally $<10 \%$ ). Superficially, this suggests a divergence between avian and mammalian systems, but detailed comparison suggests that the results may be entirely consistent: the avian data indicate that some coronary ECs arise from the PE but the fraction of ECs that originate from PE was not determined. Both avian and murine studies could therefore be interpreted to suggest that a small fraction of coronary ECs arise from PE. A recent study further pointed out that PE contains heterogeneous cell populations, and some of these subpopulations (e.g., Sema3d $d^{+}$contribute more robustly to coronary ECs than others (e.g., Tbx $18^{+}$) [7]. Some lineages traced from the PE also contributed to ECs in the SV and endocardium, providing alternative routes whereby $\mathrm{PE}$ may give rise to coronary ECs. This study did not define the fraction of coronary ECs labeled by any of these subpopulations, therefore an estimate of the extent that these additional PE subpopulations contribute to coronary ECs is currently unavailable.

Red-Horse et al. [8] recently reexamined the endothelial lining of the SV as the origin of coronary ECs. Consistent with the study by Poelmann et al. [3] in avian embryos, Red-Horse et al. observed that the first vessels of the heart tube connect to the SV. Elegant clonal labeling experiments using an EC-specific, tamoxifen-induced Cre (Cdh5-CreERT2) showed that labeling of single cells around E7.5 yielded descendant "clones" of ECs. At this point in development, $\mathrm{PE}$ cells do not express CDH5 and therefore these clones do not originate from this source. Most clones (74\%) included SV ECs. However, its relationships with extracardiac structures, such as the liver primordium, were not investigated. Interestingly, SV ECs express venous markers, but descendant ECs belong to arterial and venous lineages. Based on these data, Red-Horse et al. concluded that most coronary ECs arise by angiogenic sprouting of SV ECs onto the developing heart, where they dedifferentiate, proliferate, form the coronary plexus, and subsequently redifferentiate into coronary arteries, capillaries and veins. While these data are compelling, to what extent this mechanism contributes to the coronary vasculature cannot be determined from this study.

Wu et al. [9] used a different lineagetracing strategy to study coronary vessel origins and reached a different conclusion. This study was based on both constitutive and inducible Cre alleles driven by endocardium-specific Nfatcl regulatory elements, which do not label PE, epicardium or SV prior to E10.5. By clonal analysis, Nfatc1lineage cells differentiated to both 
artery and veins. Quantitative analysis showed that Nfatcl-labeled ECs form most intramyocardial coronary ECs (predominantly arteries) and a minority of supepicardial coronary ECs (predominantly veins). The clonal analysis of Red-Horse et al. [8] also identified endocardial budding as a source of coronary vessels. Their data showed that fewer clones (24\%) contained endocardial cells compared to $\mathrm{SV}$ cells, leading to the conclusion that endocardium makes a lesser contribution compared to the SV. However, this assumes equivalent labeling by $C d h 5$-CreERT2 under conditions where tamoxifen levels were limited. The frequency of endocardial cell labeling under these conditions may have been lower, for example if endocardial cells express lower levels of CreERT2.

Tian et al. [1] studied coronary vessel development using Apln ${ }^{\mathrm{CreERT}}$, a new lineage-tracing tool that selectively labels newly forming vessels but not established vessels or endocardium. Well-executed morphological and lineage-tracing experiments provide strong evidence that Apln ${ }^{\text {CreERT2 }}$ pulse activation at E11.5 labels nearly all subepicardial and intramyocardial coronary vessels of the ventricular free walls. Pulse labeling at this time labeled only rare ECs in the ventricular septum, suggesting that these vessels arise from ECs that express Apln ${ }^{\text {CreERT2 }}$ only after E11.5 and not from labeled ECs already present in the ventricular free walls. The endocardium appears to be an excellent candidate source for ECs in the ventricular septum. Clonal labeling experiments further demonstrated that at the single cell level, $\mathrm{Apln}^{+} \mathrm{ECs}$, named subepicardial ECs, retain the potential to differentiate into both arteries and veins.

What is the relationship between subepicardial ECs and the proposed sites of origin for coronary ECs (PE, SV, endocardium, and liver primordium)? Using in vitro organ culture, Tian et al. [1] show that these cells are generated from the SV and subsequently extend onto the ventricles. Ventricles (containing ventricular endocardium) did not generate these cells in this system, leading the authors to conclude that they arise from the SV. However, the in vitro system does not yield robust coronary vessel formation, and it is entirely possible that certain developmental processes, such as endocardial budding or epicardial differentiation, are inactive under these conditions. Thus, we can conclude that some $\mathrm{Apln}^{+} \mathrm{ECs}$ arise from SV, but the possibility of their origin also from other sources such as endocardium, PE, or liver primordium cannot be excluded.

In summary, coronary ECs arise from multiple sources, and the balance between sources likely differs by anatomic region. While many studies on coronary vessel origins appear to reach conflicting conclusions, careful considerations of the experimental approaches and their limitations suggest models consistent with most published data. For instance, perhaps endocardial budding generates most intramyocardial coronaries, while angiogenic sprouting from the SV generates most subepicardial coronaries and a subset of intramyocardial coronaries. PE cells may contribute to a fraction of both EC populations, and give rise to most of the supporting smooth muscle cells. The $\mathrm{Apln}^{+}$subepicardial ECs may represent a key common intermediate formed from all of these sources. Evaluating the contribution of each proposed cell source to this population will be important to understand the origins and growth patterns of coronary vessels. Further progress will depend on carefully quantitating the contribution of various EC sources to coronary vessel subtypes stratified by anatomic location.

Understanding the origins of coronary vessels has implications for therapeutic strategies for coronary artery diseases, as each cell source suggests distinct mechanisms. For instance, SV angiogenic sprouting would direct us to investigate the signals that induce SV EC dedifferentiation and then redifferentiation into artery and vein ECs. PE-derived ECs might be induced by

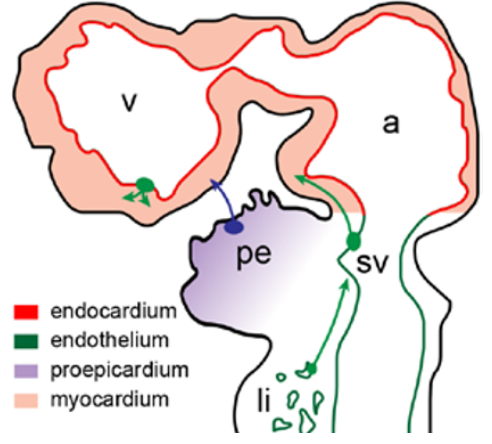

Figure 1 Diagram of E9.5 mouse embryo illustrating the proposed sources of coronary ECs. sv, sinus venosus; pe, proepicardium; li, liver primordium; v, ventricle; a, atrium.

enhancing adult epicardial EMT and EC differentiation, while an endocardial EC source would prompt us to understand the signals that regulate the endocardial budding and differentiation process. The work of Tian et al. and the many other studies summarized herein are yielding insights into the mystery of coronary vessel origins. Solving this puzzle will yield rich rewards.

\section{Bing Zhang ${ }^{1}$, William $\mathrm{T} \mathrm{Pu}^{1,2}$}

${ }^{1}$ Department of Cardiology, Boston Children's Hospital, Boston, MA 02115, USA; ${ }^{2}$ Harvard Stem Cell Institute, Harvard University, Cambridge, MA 02138, USA

Correspondence: William T Pu

E-mail: wpu@pulab.org

\section{References}

1 Tian X, Hu T, Zhang H, et al. Cell Res 2013; 23:1075-1090.

2 Mikawa T, Fischman DA. Proc Natl Acad Sci USA 1992; 89:9504-9508.

3 Poelmann RE, Gittenberger-de Groot AC, Mentink MM, et al. Circ Res 1993; 73:559568.

4 Wilm B, Ipenberg A, Hastie ND, et al. Development 2005; 132:5317-5328.

5 Zhou B, Ma Q, Rajagopal S, et al. Nature 2008; 454:109-113.

6 Cai CL, Martin JC, Sun Y, et al. Nature 2008; 454:104-108.

7 Katz TC, Singh MK, Degenhardt K, et al. Dev Cell 2012; 22:639-650.

8 Red-Horse K, Ueno H, Weissman IL, et al. Nature 2010; 464:549-553.

9 Wu B, Zhang Z, Lui W, et al. Cell 2012; 151:1083-1096. 